THE CONTRIBUTION OF HIPPARCOS TO THE STUDY OF THE STELLAR METAL-RICH POPULATION IN THE SOLAR NEIGHBOURHOOD

\author{
G. CAYREL de STROBEL ${ }^{1}$, C. SOUBIRAN ${ }^{2}$, Y. LEBRETON ${ }^{1}$ \\ 1 Observatoire de Paris, DASGAL/URA CNRS 335, 92195 Meudon Cedex, France \\ ${ }^{2}$ Observatoire de Bordeaux, BP 89, 33270 Floirac, France
}

The '1996 Edition' of the Catalogue of $[\mathrm{Fe} / \mathrm{H}]$ determinations by Cayrel de Strobel et al. (1997, A\&A S 124,1) and two recent papers by Castro et al. (1997, AJ Vol.114, N.1) and by Feltzing and Gustafsson (A\&A in press) have made possible to increase in the theoretical HR diagram $\left(\log \mathrm{T}_{\text {eff }}\right.$, $\left.\mathrm{M}_{b o l}\right)$ the number of SMR stars.

The SMR Stars of this new enlarged sample had to have reliable absolute magnitudes, coming all from Hipparcos parallaxes, precise bolometric corrections, effective temperatures and metal abundances from high resolution detailed spectroscopic analyses.

With the help of an appropriate grid of isochrones computed by Lebreton (1997, Perryman et al. A\&A, in press), 'turn-off ages' could then be attributed to the slightly evolved stars (subgiants) of the sample. The $\left(\log \mathrm{T}_{e f f}, \mathrm{M}_{b o l}\right.$ ) diagram constituted by the new sample of SMR stars, shows that the conclusions in a former paper by Cayrel de Strobel $(1987$, A\&AJ 8,141) remain valid: the SMR stars are old stars in spite of their higher than solar metallicity. The result, that metal-rich stars were in the mean old stars, was interpreted in the 1987 paper as due to a more chemical uniformity of the nowadays interstellar medium of the Galaxy with respect of the older much more active interstellar medium.

\title{
THE ABSOLUTE MAGNITUDE OF THE EARLY-TYPE MK STANDARDS FROM HIPPARCOS PARALLAXES
}

\author{
C. JASCHEK ${ }^{1}$, A.E. GÓMEZ ${ }^{2}$ \\ 1 Teso de la Feria, 39, E37008 Salamanca, Spain \\ 2 Observatoire de Paris, DASGAL/CNRS URA 335, 92195 Meudon Cedex, France
}

We have analysed the standards of the MK system in the B0-F5 spectral region with the help of Hipparcos parallaxes, using only stars for which the error on the absolute magnitude is $\leq 0.3$ mag. The sample stars (about one hundred) were scrutinized for companions and for interstellar extinction. We find that the main sequence is a wide band and that, although in general giants and dwarfs have different absolute magnitudes, the separation between luminosity class V and III is not clear. We conclude that there is no strict relation between luminosity class and absolute magnitude. The relation is only a statistical one and has a large intrinsic dispersion.

We have analysed similarly the system of standards defined by Garrison and Gray (1994) separating low and high rotational velocity standards. We find similar effects as in the original MK system.

\section{References}

Garrison, R.F., Gray, R.O. (1994) AJ, Vol.104, pp. 1556-1564 